\title{
MATURATION OVOCYTAIRE APRÈS STIMULATION FOLLICULAIRE CHEZ LA GÉNISSE IMPUBÈRE
}

\author{
J. TESTART et J. ARRAU * \\ avec la collaboration technique de P. Chesne \\ Station centrale de Physiologie animale, I. N.R. A., \\ 78350 Jouy en Josas (France)
}

\section{RÉSUMÉ}

Nous avons provoqué avec PMSG la croissance folliculaire préovulatoire chez des Veaux impubères, porteurs ou non d'une éponge vaginale imprégnée de FGA, et nous avons étudié la reprise de la méiose dans les ovocytes.

L'injection de PMSG est suivie, I6 heures plus tard, d'une nette augmentation de taille des plus gros follicules. Cette augmentation est stoppée entre I et 3 jours après PMSG et reprend le quatrième jour.

Chez les animaux traités avec FGA, le retrait de l'éponge vaginale, le $5^{\mathbf{e}}$ jour après PMSG, est suivi 16 heures plus tard, d'une ultime croissance des plus gros follicules.

La reprise de la méiose est observée dans des follicules de toutes tailles; alors que le quart des ovocytes observés 2 à 4 jours après l'injection de PMSG seule, ont atteint ou dépassé le stade métaphase I, ce stade n'est pas atteint avant le $5^{\mathbf{e}}$ jour chez les animaux traités avec FGA et apparaît massivement le jour qui suit le retrait de l'épcnge.

La relation entre rupture de l'état dictyé et dégénérescence folliculaire ainsi que les stimulations endocrines en cause dans les phénomènes observés, sont discutées.

De nombreux travaux depuis CASIDA et al. (I943), MARDEN (I953) et BLACK et al. (I953) rapportent les tentatives faites pour obtenir des œufs en grand nombre chez le Veau impubère. La bibliographie du sujet a été établie par HaFEz (I970).

La plupart des auteurs signalent l'inconvénient présenté par l'étalement dans le temps des ovulations provoquées et le faible rendement des essais de fécondation. Cependant, le problème présente un intérêt considérable si on envisage de transplanter des œufs fécondés dans des nourrices utérines (FooTE et ONUMA, I970; SEIDEL, et al., I97I).

* Adresse permanente : Laboratorio de Embriologia, Instituto de Ciencias Biologicas, Universidad Católica. Santiago de Chile (Chili). 
Nous avons montré la possibilité de synchroniser les ovulations provoquées par un traitement progestagène (TESTART, I972 $a$ ). Le présent travail a pour but d'étudier la reprise de la méiose dans l'ovocyte durant la croissance des follicules stimulés par la gonadotropine sérique.

\section{MATÉRIEL ET MÉTHODES}

Quarante-neuf veaux femelles de races $F . F . P . N$. et Normande, âgés de 3 à 4 mois (poids : 120 à $150 \mathrm{~kg}$ ) et nourris exclusivement au lait artificiel sont divisés en deux groupes.

Tous reçoivent une injection intramusculaire de I 800 à 3000 UI de gonadotropine sérique ("Intervet"). Pour 22 d'entre eux (de race F.F.P.N.) on procède, le même jour, à la pose d'une éponge vaginale imprégnée avec $60 \mathrm{mg}$ d'acétate de fluorogestone (FGA "Searle ") ; cette éponge est laissée en place pendant 4 jours (TESTART, 1972 a).

Les animaux recevant seulement PMSG sont abattus entre 8 et go heures après l'injection. Ceux dont le traitement comporte FGA sont abattus entre 96 et I68 heures après l'injection de PMSG, soit de o à 72 heures après le retrait de l'éponge.

Au moment de l'abattage, les ovaires sont prélevés rapidement, pesés et décrits. On dénombre les follicules faisant saillie à la surface de l'ovaire et on les mesure à l'aide d'une échelle millimétrée avant de prélever les ovocytes par aspiration dans les follicules avec une pipette de verre. Ces ovocytes sont ensuite recherchés sous loupe binoculaire dans une solution de citrate de sodium à 3,5 p. 100 (pression osmotique : 280 mosmol), à température ambiante ; cette technique permet d'éviter la coagulation du liquide folliculaire pendant la recherche des ovocytes.

Le délai entre l'abattage de l'animal et la fixation de tous les ovocytes récupérés n'excède jamais 30 minutes.

Les follicules inférieurs à $3 \mathrm{~mm}$ des animaux traités avec PMSG + FGA sont isolés des ovaires et fixés in toto pour étude histologique.

Puis on réalise des préparations histologiques selon la méthode de C. Thibault (I949); les coupes de ro $\mu$ d'épaisseur sont observées à l'aide d'un objectif à immersion ( $\times$ Ioo).

On a classé les images montrées par le matériel nucléaire de l'ovocyte en 3 catégories :

- dictyé : noyau généralement central dans le cytoplasme avec nucléole très coloré et chromatine diffuse ;

- prémétaphase : noyau souvent périphérique avec nucléole pâle, chromatine progressivement plus condensée, membrane nucléaire moins nette ;

- stades plus avancés depuis métaphase I jusqu'à métaphase II. Le stade ultime de la prémétaphase $\left(\mathrm{PM}_{4}\right)$ a parfois été confondu dans la représentation graphique avec les stades plus avancés (fig. 2). Ce stade est caractérisé selon THIBAuLr (communication personnelle) par la condensation extrême du matériel nucléaire figurant une masse chromatique compacte, périphérique dans le cytoplasme et encore entourée d'un reliquat de membrane nucléaire, le nucléole ayant disparu.

L'ovocyte est considéré comme dégénérescent quand le cytoplasme apparaît hétérogène après coloration à l'éosine avec parfois de nombreuses et larges vacuoles. Le cumulus oophorus, entraîné par aspiration de l'ovocyte, est considéré comme picnotique quand il y a plus de quelques cellules picnotiques isolées.

\section{RÉSULTATS}

\section{A. - Croissance folliculaire préovulatoire}

a) Veaux traités avec PMSG seule.

La figure I montre l'évolution du diamètre moyen des 3 plus gros follicules. Celui-ci, qui est de 3,8 à 4,6 mm 8 à I2 heures après l'injection de PMSG s'accroît brusquement $(P<0,5)$ à partir de $\mathrm{I} 6$ heures $(7, \mathrm{I} \pm \mathrm{I}, 2 \mathrm{~mm})$ puis il n'augmente plus jusqu'à 72 heures $(7, \mathrm{I} \pm \mathrm{I}, \mathrm{I} \mathrm{mm})$ et croît de nouveau brusquement $(\mathrm{P}<0, \mathrm{I})$ pour atteindre $13,2 \mathrm{~mm}$ à 90 heures. 
On constate que l'ovulation apparaît depuis 3 jours après PMSG pour une proportion limitée d'animaux (I/3 à 72 heures; $3 / 8$ à 90 heures); de plus, dans ces conditions, on obtient qu'une seule ovulation par animal.

PMSG seule

PMSG +FGA

E

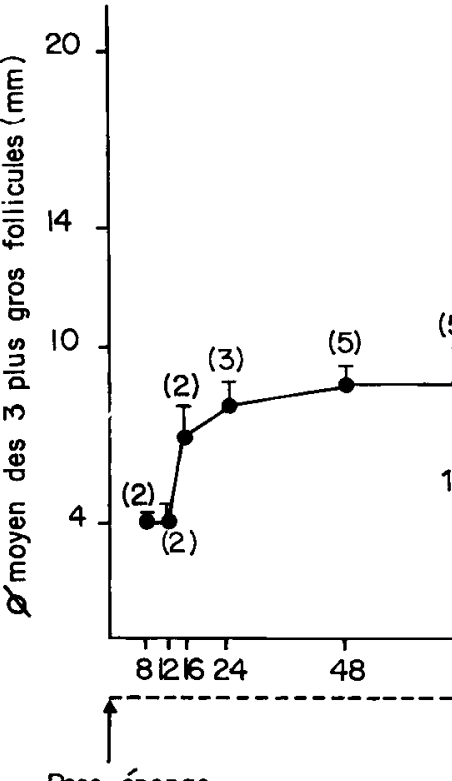

Pose éponge

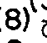

(4)
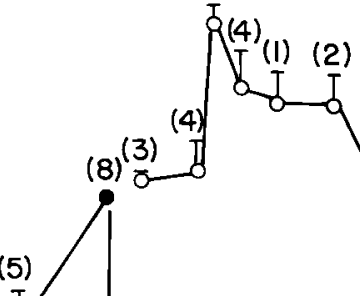

(5)

(8)

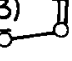

(5)
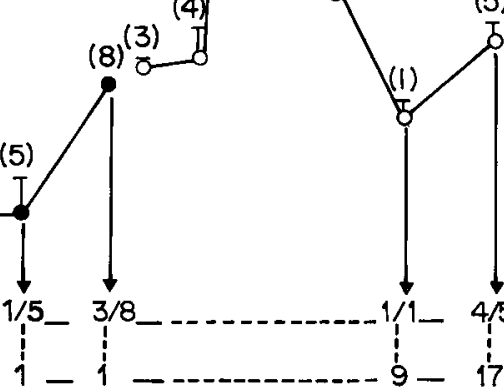

onimoux

qui ovulent

nombre moyen

d'ovulations

heures après injection PMSG

$\sqrt{12162028} 40 \quad 54 \quad 72$

heures après retrait éponge

FIG. I. - Evolution du diametre moyen des trois plus gros follicules

- Traitement des veaux avec PMSG seule

- Traitement des veaux avec PMSG + FGA (éponge vaginale)

( ) Nombre d'animaux

TABLEAU I

Croissance folliculaire après l'injection de PMSG ou l'arrêt de FGA

\begin{tabular}{|c|c|c|c|}
\hline $\begin{array}{c}\text { Intervalle } \\
\text { PMSG/abattage } \\
\text { (heures) }\end{array}$ & Nombre de veaux & $\begin{array}{l}\text { Nombre de veaux } \\
\text { avec follicules } \\
>4 \mathrm{~mm}\end{array}$ & $\begin{array}{l}\text { Nombre moyen } \\
\text { de follicules } \\
>4 \mathrm{~mm}\end{array}$ \\
\hline $\begin{array}{c}<16 \\
16 \text { a } 24\end{array}$ & $\begin{array}{l}4 \\
5\end{array}$ & $\begin{array}{l}2 \\
5\end{array}$ & $\begin{array}{r}1,2 \\
12,2\end{array}$ \\
\hline $\begin{array}{c}\text { Intervalle } \\
\text { Arrêt FGA/ } \\
\text { abattage } \\
\text { (heures)* }\end{array}$ & Nombre de veaux & $\begin{array}{c}\text { Nombre de veaux } \\
\text { avec follicules } \\
>14 \mathrm{~mm}\end{array}$ & $\begin{array}{l}\text { Nombre moyen } \\
\text { de follicules } \\
>14 \mathrm{~mm}\end{array}$ \\
\hline $\begin{array}{c}<16 \\
16 \text { à } 24\end{array}$ & $\begin{array}{l}7 \\
6\end{array}$ & $\begin{array}{l}2 \\
6\end{array}$ & $\begin{array}{l}0,8 \\
4,3\end{array}$ \\
\hline
\end{tabular}

* Traitement FGA de 4 jours commencé en même temps que PMSG. 
b) Veaux traités avec $P M S G+F G A$.

Au moment du retrait de l'éponge vaginale (soit 4 jours après l'injection gonadotrope), le diamètre moyen des 3 plus gros follicules (fig. I) est de $13,6 \pm 0,33 \mathrm{~mm}$ et n'est pas différent $\mathrm{I} 2$ heures plus tard (I4,0 $\pm \mathrm{I}, 0 \mathrm{~mm})$. C'est après que ce diamètre moyen augmente $(P<0, I)$ rapidement pour atteindre $I 9,0 \pm I, 4 \mathrm{~mm}$ à $\mathrm{I} 6$ heures, puis on observe une diminution ( $\mathrm{r} 4,5 \pm 0,67 \mathrm{~mm}$ à 72 heures) due à 1'élimination des plus gros follicules transformés en corps jaunes. En effet, la presque totalité des animaux (4/5) ont ovulé et le nombre moyen d'ovulations ( $\mathrm{I} 7,0)$ est important.

Le tableau I compare les effectifs des plus gros follicules avant et après I6 heures suivant l'injection de PMSG ou le retrait de l'éponge : c'est en effet de I6 à 24 heures après PMSG que le nombre moyen de follicules supérieurs à $4 \mathrm{~mm}$, auparavant réduit $(\mathrm{I}, 2)$ est décuplé $(\mathrm{I} 2,2)$. De même, c'est $\mathrm{I} 6$ heures après le retrait de l'éponge que le nombre moyen de follicules supérieurs à $\mathrm{I} 4 \mathrm{~mm}$ s'accroît très nettement (de 0,8 à 4,3 ).

\section{B. - Maturation ovocytaire}

a) Veaux traités avec PMSG seule.

Comme le montre la figure 2 , le taux d'ovocytes en cours de maturation évolue peu pendant les 4 jours qui suivent l'injection gonadotrope : $2 \mathrm{I}, 5 \mathrm{p}$. IoO à 2 jours, 26,7 p. I0o à 3 jours, 28,8 p. Ioo à 4 jours. En même temps, le taux de noyaux dictyés montre seulement une légère diminution (de 42,9 p. IOo à $32,7 \mathrm{p}$. IO0) ; la fréquence de rupture de la vésicule germinative est donc légèrement évolutive.

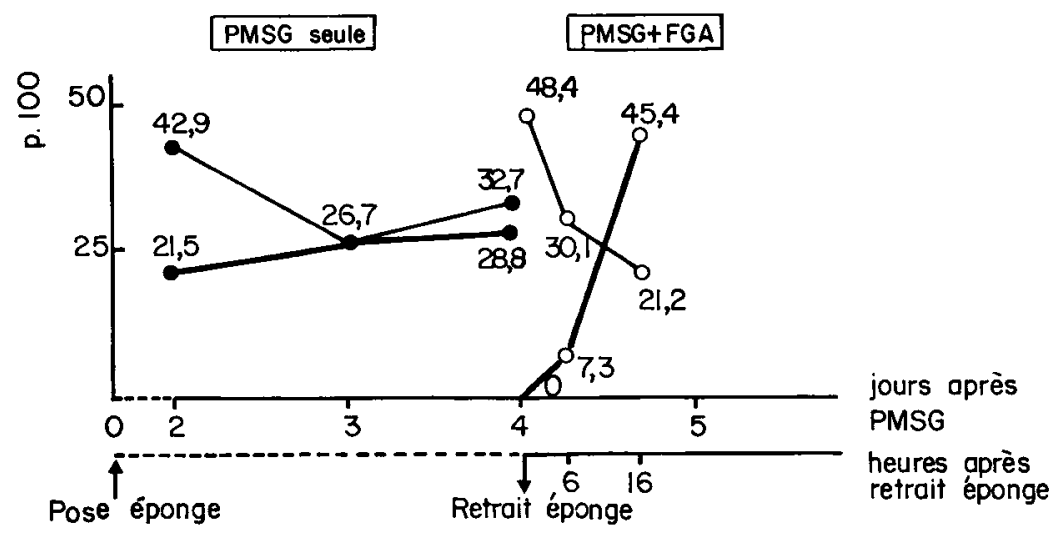

FIG. 2. - Evolution du noyau de l'ovocyte après traitement gonadotrope

p. Ioo noyaux dictyés

- Traitement des veaux avec PMSG seule

- Traitement des veaux avec PMSG + FGA (éponge vaginale)

b) Veaux traités avec $P M S G+F G A$.

Alors qu'aucun ovocyte n'a dépassé la prophase méiotique au moment du retrait de 1'éponge (fig. 2), très rapidement le taux de noyaux présentant divers stades de maturation depuis la fin de prémétaphase $\left(\mathrm{PM}_{4}\right.$, voir Matériel et méthodes) 
augmente $(7,3$ p. Ioo 6 heures plus tard) pour atteindre 45,4 p. Ioo $(6$ à 20 heures après le retrait de l'éponge $(P<0, I)$.

Le petit nombre d'ovocytes observés après plus de 20 heures suivant le retrait de l'éponge ne permet pas de conclure, cependant on observe une tendance à la diminution du taux de noyaux en cours de maturation : I9 sur 69 , soit $27,5 \mathrm{p}$. Ioo entre 40 et 44 heures après le retrait de l'éponge, c'est-à-dire juste avant l'ovulation.

L'évolution de la maturation ovocytaire en fonction de la taille du follicule (fig. 3) montre une chute progressive du taux de noyaux dictyés $(45,6 \mathrm{p}$. Ioo dans les follicules inférieurs à $3 \mathrm{~mm}$, contre $2 \mathrm{I}, 7 \mathrm{p}$. Ioo dans les follicules supérieurs à I4 $\mathrm{mm}$ ) et de celui des ovocytes en prémétaphase (48 p. Ioo contre $26, \mathrm{I}$ p. Ioo respectivement), au profit des ovocytes entrés en métaphase $(6,4 \mathrm{p}$. Ioo contre 52,2 p. Ioo respectivement).

L'évolution de la maturation ovocytaire selon le délai depuis le retrait de l'éponge vaginale est parallèle; elle souligne l'absence de noyaux en métaphase sous l'effet de FGA mais aussi la présence abondante $(5 \mathrm{I}, 6 \mathrm{p}$. Ioo) d'ovocytes montrant les premiers signes de rupture de la vésicule germinative à ce moment.

La figure 3 montre, pour chaque état nucléaire, la fréquence des dégénérescences cytoplasmiques de l'ovocyte ou des picnoses du cumulus. Celles-ci sont toujours plus importantes dans les follicules dont le noyau est avancé dans sa maturation puisqu'elles concernent la moitié des ovocytes en métaphase, quels que soient la taille du follicule ou le délai depuis le retrait de l'éponge.

(a) en fonction de la taille du follicule
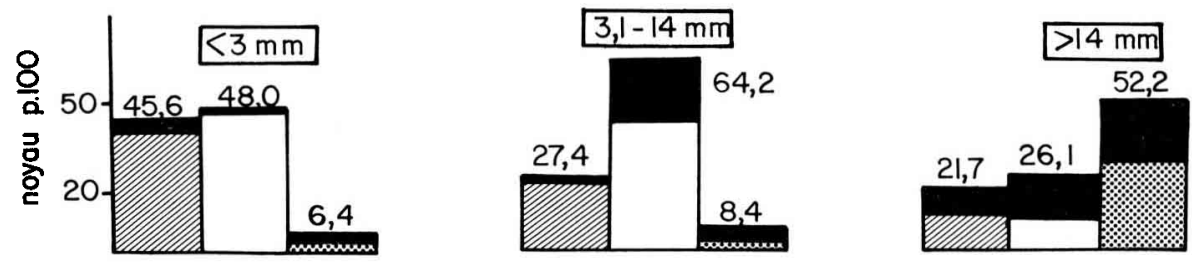

(b) en fonction de l'intervalle, retrait de l'éponge (FGA)-abattage
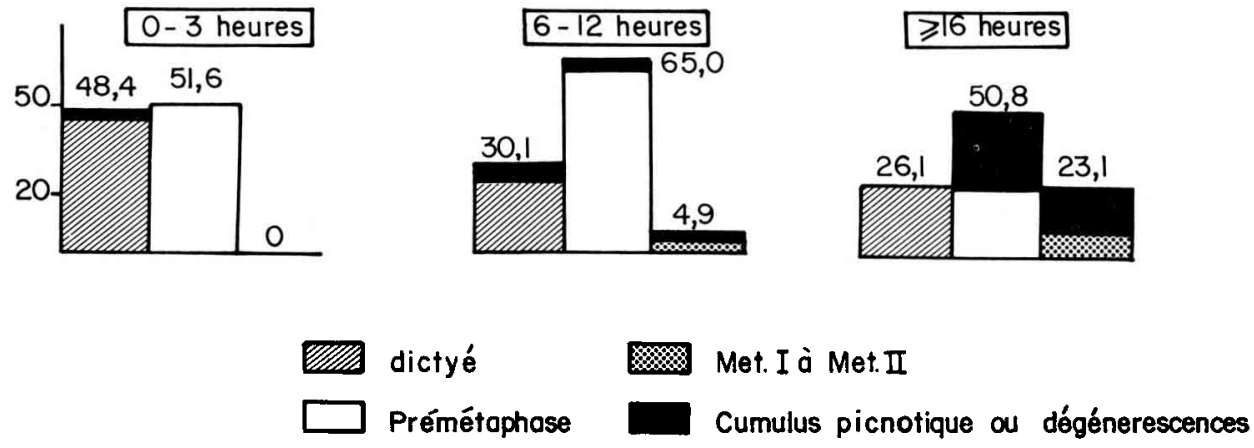

FIG. 3. - Stades de maturation ovocytaire après traitement PMSG-FGA (éponge vaginale posée en même temps que PMSG, retirée après 4 jours) 


\section{DISCUSSION}

\section{A. - Croissance folliculaire préovulatoire}

La présence fréquente chez le Veau impubère d'un gros follicule préexistant à tout traitement gonadotrope retire toute valeur à l'expression du développement folliculaire par le diamètre du plus gros follicule. Par ailleurs, la variabilité individuelle de l'effectif folliculaire relativise la réponse à PMSG mesurée par le nombre de follicules correspondant à une classe de taille déterminée. Aussi, il nous a paru préférable de considérer le diamètre moyen d'un nombre constant de gros follicules ; nous avons arbitrairement fixé ce nombre à 3 .

Comme il ressort de la figure I, nous n'avons pas effectué l'abattage d'animaux porteurs d'une éponge vaginale dans les 4 jours qui suivent l'injection de PMSG ; cependant, la courbe de croissance folliculaire que nous obtenons depuis le retrait de l'éponge est complémentaire de celle observée pour les jours qui suivent 1'injection de PMSG seule. Aussi, nous supposons que la réponse folliculaire à la stimulation gonadotrope n'est pas modifiée par la présence du progestagène, au moins pour le niveau de développement atteint 4 jours plus tard.

Cette réponse folliculaire montre donc 3 phases successives d'accroissement : dans la seconde moitié du premier jour après l'injection gonadotrope, puis du troisième au quatrième jour, enfin, au moins dans le cas du traitement progestagène utilisé, à la fin du cinquième jour, c'est-à-dire dans la seconde moitié du jour qui suit l'arrêt du progestagène.

Chez la Vache cyclique, Marion, GiER et Choudary (I968) ne trouvent pas de phases d'accélération dans la croissance folliculaire entre $\mathrm{I}$ et $12 \mathrm{~mm}$, mais seulement une croissance plus rapide durant les dernières 24 heures avant l'ovulation. Le follicule préovulatoire atteint ainsi $\mathrm{r} 6$ à $\mathbf{I} 8 \mathrm{~mm}$, taille qui n'est atteinte, dans notre expérience, que chez les Veaux traités avec FGA.

La superovulation qui survient 2 jours après le retrait de l'éponge est responsable de la diminution du diamètre moyen des 3 plus gros follicules persistant sur les ovaires. Contrairement à ce qui se passe avec de faibles doses de PMSG (I25 UI tous les 2 jours ; TESTART, $1972 b$ ) le FGA, dans les conditions de notre expérience, n'inhibe pas la croissance folliculaire provoquée.

\section{B. - Maturation ovocytaire}

L'évolution nucléaire n'est pas parallèle à la croissance folliculaire; après PMSG seule, le taux d'ovocytes, en fin de prémétaphase $\left(\mathrm{PM}_{4}\right)$ et au-delà, reste sensiblement identique du $2^{e}$ au $4^{e}$ jour; de même après PMSG-FGA puisque ce taux est encore nul après 4 jours. Contrairement aux conclusions de HAFEZ et ISHIBASHI (I964), chez la Vache cyclique, nous avons observé la maturation nucléaire complète (jusqu'à la métaphase II) d'ovocytes inclus dans des follicules très petits, $\mathrm{y}$ compris inférieurs à $3 \mathrm{~mm}$.

Cependant, la brusque augmentation de la taille folliculaire qui suit le retrait 
de l'éponge vaginale est accompagnée fréquemment de la maturation nucléaire. Cette image doit être nuancée puisque avant même le retrait de l'éponge vaginale, plus de la moitié des ovocytes ont entamé le processus de rupture de la vésicule germinative (5I,6 p. Ioo d'ovocytes en prémétaphase, fig. 3 ).

Le délai d'apparition du stade Métaphase II, rapporté pour l'ovocyte bovin cultivé in vitro par EDWARds (I965), Foote et ThIBAulT (I969), HuNTER (I97I), est d'environ 24 à $3 \mathbf{I}$ heures après la mise en culture. Nous observons des images de métaphase I depuis 6 heures après le retrait de l'éponge vaginale, ce qui correspond effectivement à la rupture de l'état dictyé le $4^{\mathrm{e}}$ jour après PMSG.

Foote et Thrbault (I969) ont analysé le rôle des cellules folliculaires dans le maintien de l'état dictyé de l'ovocyte et montré qu'une picnose même localisée de la granulosa est suffisante pour lever cette inhibition. Or, l'importance de la dégénérescence $d u$ follicule ne peut être estimée valablement que par examen histologique du follicule entier; nous n'avons encore pratiqué cet examen que pour les follicules inférieurs à $3 \mathrm{~mm}$ des animaux traités avec PMSG + FGA, et nous n'avons observé la maturation nucléaire que dans des follicules dont l'aspect histologique est anormal (picnose, disparition de la membrane basale). L'évolution nucléaire décrite ici devra être précisée après examen de gros follicules dans lesquels se produit couramment la maturation ovocytaire. Le fait que seulement la moitié des ovocytes ovulés se divisent normalement après insémination artificielle, selon nos essais en cours, peut être rapproché du taux élevé de follicules picnotiques (cumulus seul observé) ou d'ovocytes à cytoplasme dégénérescent, montrés dans la figure 3 .

Quelles que soient les causes mécaniques de la rupture de la vésicule germinative et leurs conséquences sur la normalité de l'ovocyte, il reste à analyser l'origine endocrine d'une telle évolution.

Certainement, l'injection gonadotrope est responsable de la rupture de l'état dictyé constatée 2 à 4 jours plus tard pour le quart environ des ovocytes observés. Il ne s'agit pas d'une action directe de PMSG sur l'ovaire puisqu'elle n'est pas retrouvée chez les animaux soumis au progestagène.

Une seconde stimulation nucléaire doit prendre place 3 à 4 jours après l'injection de PMSG et n'est pas empêchée par FGA puisque la moitié des ovocytes observés avant le retrait de l'éponge montrent des noyaux en prémétaphase. On peut rapprocher cette vague d'évolution nucléaire de la vague de croissance folliculaire constatée 3 jours après PMSG et, envisager l'action d'un haut niveau œestrogénique atteint à ce moment sur une décharge gonadotrope que FGA est impuissant à inhiber. Peilietier et Thrmonier (I969) ont montré, chez la Brebis, que FGA empêche la décharge cyclique de $\mathrm{LH}$; les dosages radioimmulogiques effectués sur le plasma des Veaux ('TESTART et KANN, à paraître) montrent que le niveau plasmatique de LH est inférieur à $0,3 \mathrm{ng} / \mathrm{ml}$ durant tout le traitement avec FGA. Ce niveau atteint plusieurs dizaines de $\mathrm{ng} / \mathrm{ml}$ lors d'un pic situé de $\mathrm{I}_{5}$ à 20 heures après le retrait de l'éponge vaginale, c'est-à-dire quand un grand nombre d'ovocytes ont déjà atteint le stade métaphase I ou II. Un tel niveau plasmatique de $\mathrm{L} H$ doit être impliqué plutôt dans le processus ovulatoire qui suit que dans la maturation nucléaire qui précède, mais on ne peut négliger le rôle éventuel d'un très faible niveau de base plasmatique de LH.

Par ailleurs, la reprise de la méiose observée avant le pic sanguin de $\mathrm{LH}$ ne correspond pas nécessairement à la maturation préovulatoire des ovocytes, mais 
n'est peut-être que le reflet de l'atrésie folliculaire. En effet, le délai entre ce pic de $\mathrm{LH}$ et l'ovulation (au minimum 30 heures) est suffisant pour permettre la maturation d'une autre population ovocytaire destinée à ovuler.

\section{SUMMARY}

\section{OOCYTE MATURATION FOLLOWING FOLLICLE STIMULATION}

IN THE CALF

Follicle growth and resumption of meiosis is studied in $493-4$ month-old $F . F$. $P$. $N$. and Norman heifers following gonadotrophic stimulation (I 500-3 000 IU of PMSG). The same day as PMSG injection, vaginal sponges impregnated with $60 \mathrm{mg}$ of fluorogestone acetate (FGA) are put into 22 heifers and left in place for 4 days.

Animals receiving only PMSG are slaughtered in the 4 days after injection. Those treated with FGA are slaughtered between days 5-8, or 3 days after the sponge is removed. If ovulation occurs within 3 days after PMSG, only one follicle per animal is involved. FGA prevents ovulation up to day 7 after PMSG (day 3 after sponge removal), but many ovulations are obtained (average 9-I 7 , fig. I).

The average diameter of the three largest follicles $(3.8-4.6 \mathrm{~mm})$ is almost doubled $\mathrm{I} 6$ hours after PMSG (7.I mm), and only increases again after day 3 (13.2 mm, 90 hours after PMSG).

In FGA-treated animals, the average diameter of the three largest follicles ( $14.0 \mathrm{~mm}$ when sponge is removed 96 hours after PMSG) reaches $19.0 \mathrm{~mm}$ sixteen hours later (fig. I).

One quarter of the ovocyte nuclei examined 2-4 days after PMSG reach or exceed metaphase I, but in FGA-treated animals, this stage is only reached after the sponge is removed, although the nucleus shows signs of activation (premetaphase) before interruption of the FGA treatment (fig. 2).

Although ovocyte maturation occurs more frequently in the large follicles (fig. 3), resumption of meiosis, and even complete nuclear evolution (metaphase II), is observed in follicles smaller than $3 \mathrm{~mm}$ diameter.

The relation between dictyate stage rupture and follicular degeneration, as well as endocrine stimulation, are discussed.

\section{RÉFÉRENCES BIBLIOGRAPHUQUES}

Black W. G., Ulberg L. C., Christian R. E., Casida L. E., I953. Ovulation and fertilization in the hormone-stimulated calf. J. Dairy Sci., 36, 274.

Casida L. E., Meyer R. K., McShan W. H., Wisnicky W., I943. Effects of pituitary gonadotrophins on the ovaries and the induction of superfecundity in cattle. Am.J.Vet. Res., 4, 76.

Edwards R. G., 1965. Maturation in vitro of mouse, sheep, cow, pig, rhesus monkey and human ovarian oocytes. Nature, 208, 349-35I.

Foote R. H., ONuma H., r97o. Superovulation, ovum collection, culture and transfer. J. Dairy Sci., 53, I68I.

Foote W. D., Thibault C., 1969. Recherches expérimentales sur la maturation in vitro des ovocytes de Truie et de Veau. Ann. Biol. anim. Bioch. Biophys., 9, 329-349.

Hafez E. S. E., I970. Superovulation and preservation of mammalian egg. Acta. Endocr., 62, suppl. I $40,44 \mathrm{p}$.

Hafez E. S. E., Ishibashi I., I964. Maturation division in bovine oocytes following gonadotrophin injections. Cytogenetics, 3, $167-83$.

Hunter R. H., Lawson R. A. S., Rowson L. E. A., 1972. Maturation, transplantation and fertilization of ovarian oocyte in cattle. J. Reprod. Fertil., 30, 325.

Marden W. G. R., I953. The hormone control of ovulation in the calf. J. Agric. Sci., 43, $38 \mathrm{r}-406$.

Marion G. B., Gier H. T., Choudary J. B., I968. Micromorphology of the bovine ovarian follicular system. J. Anim. Sci., 27, 45 I-465. 
Pelletier J., Thimonier J., ig69. Étude de la décharge ovulante par dosage radioimmunologique de la LH plasmatique chez la Brebis normale ou traitée par un progestagène. C. R. Acad. Sci., 268, 573-576.

Seidel G. E., Larson L. L., Spilman C. H., Hahn J., Foote R. H., i97i. Culture and transfer of calf ova. J. Dairy Sci., 54, 923-925.

Testart J., r972a. Synchronisation des ovulations induites chez le Veau impubère. VII Congr. Reprod. anim. Insém. artif., Munich.

TESTART J., I972 b. Réponse folliculaire du Veau femelle impubère à divers traitements de gonadotropine sérique associée ou non à un progestagène. Ann. Biol. anim. Bioch. Biophys., 12, 397-409.

Thibault C., I949. L'auf des Mammifères. Son développement parthénogénétique. Thèse Doct. Sci., Fac. Sci., Paris, Masson, p. 219. 